Article

\title{
Development and Characterization of Two-Dimensional Gratings for Single-Shot X-ray Phase-Contrast Imaging
}

\author{
Margarita Zakharova ${ }^{1, *(\mathbb{D})}$, Vitor Vlnieska ${ }^{1}$, Heike Fornasier ${ }^{1}$, Martin Börner ${ }^{1}$, \\ Tomy dos Santos Rolo ${ }^{2}$ (D), Jürgen Mohr ${ }^{1}$ and Danays Kunka ${ }^{1}$ (i) \\ 1 Karlsruhe Institute of Technology (KIT), Institute of Microstructure Technology (IMT), \\ Hermann-von-Helmholtz-Platz 1, 76344 Eggenstein-Leopoldshafen, Germany; vitor.vlnieska@kit.edu (V.V.); \\ heike.fornasier@kit.edu (H.F.); martin.boerner@kit.edu (M.B.); juergen.mohr@kit.edu (J.M.); \\ danays.kunka@kit.edu (D.K.) \\ 2 Karlsruhe Institute of Technology (KIT), Institute of Photon Science and Synchrotron Radiation (IPS), \\ Herrmann-von-Helmholtz-Platz 1, 76344 Eggenstein-Leopoldshafen, Germany; tomy.rolo@gmail.com \\ * Correspondence: margarita.zakharova@partner.kit.edu; Tel.: + 49-721-608-26838
}

Received: 22 December 2017; Accepted: 16 March 2018; Published: 18 March 2018

Featured Application: The implementation of single-shot grating-based imaging within existing X-ray scanners will offer enhanced imaging contrast for weakly absorbing objects and materials with similar absorption cross-sections, profiting from phase and small-angle scattering contrasts. Due to the provided multi-modal information, such techniques can find broad applications in pre-clinical imaging of soft tissues, the determination of composite material structural orientation, and the visualization of formation processes of different natures, etc. To adapt the imaging modality to the intended application and profit from the versatility of those techniques, one requires customized optical elements. Our group has developed novel two-dimensional gratings, optimized for X-ray imaging at energies from $8 \mathrm{keV}$ up to $25 \mathrm{keV}$, which can be used for orientation-independent inspection of materials within radiography setup.

Abstract: Single-shot grating-based phase-contrast imaging techniques offer additional contrast modalities based on the refraction and scattering of $\mathrm{X}$-rays in a robust and versatile configuration. The utilization of a single optical element is possible in such methods, allowing the shortening of the acquisition time and increasing flux efficiency. One of the ways to upgrade single-shot imaging techniques is to utilize customized optical components, such as two-dimensional (2D) X-ray gratings. In this contribution, we present the achievements in the development of $2 \mathrm{D}$ gratings with UV lithography and gold electroplating. Absorption gratings represented by periodic free-standing gold pillars with lateral structure sizes from $5 \mu \mathrm{m}$ to $25 \mu \mathrm{m}$ and heights from $5 \mu \mathrm{m}$ to $28 \mu \mathrm{m}$ have shown a high degree of periodicity and defect-free patterns. Grating performance was tested in a radiographic setup using a self-developed quality assessment algorithm based on the intensity distribution histograms. The algorithm allows the final user to estimate the suitability of a specific grating to be used in a particular setup.

Keywords: 2D gratings; UV lithography; grating-based X-ray imaging; single-shot phasecontrast imaging

\section{Introduction}

Phase-contrast imaging methods have attracted a lot of attention in the scientific community due to their ability to offer enhanced information on the inner structure of the various objects, in addition 
to conventional attenuation contrast [1,2]. These methods enable the detection of X-ray refraction and scattering while passing through the object under investigation. Following increasing interest, the application of phase-contrast imaging was made available not only at synchrotron facilities with highly coherent and monochromatic radiation, but also with conventional X-ray tubes [1]. X-ray refraction and scattering within laboratory setups are usually detected with the help of the intended optical components, so-called gratings, introduced into the beam path. Several interferometric [2-4] and non-interferometric [5-7] techniques utilizing grating structures have been proposed.

Despite the remarkable results in imaging achieved by these techniques, there is still an open question as to whether it is possible to further optimize the stability of the setup, acquisition time, and flux efficiency [8]. From this perspective, one of the most promising approaches is the utilization of a single optical element in a single-shot configuration. The implementation of only one optical component in the beam path relaxes the alignment requirements, increases the mechanical stability and versatility of the setup, and significantly reduces the attenuation introduced by the grating. Single-shot acquisition of imaging information, in turn, substantially shortens the total exposure time, simplifies the experimental procedure and reduces the total amount of data to be processed. All those advantages can be exploited by non-interferometric single-exposure imaging methods [9-11], in which all three contrast modalities are retrieved from a single projection image. In this approach, the incident beam is periodically modulated by the absorption grating, which can be placed before or after the object. The projected grating pattern distortion introduced by the object is recorded with a single exposure and then analyzed [6,9-15].

To respond to the growing interest in the single-shot phase-contrast imaging, one should pay special attention to the optical components in use. Gratings for X-ray imaging are represented by a matrix with periodically-alternating opaque and transparent structures. When $\mathrm{X}$-ray radiation is incident on the grating, it is partially transmitted by the low-absorbing substrate, while radiation incident on the gold structures' array is predominantly absorbed. Diffraction on the multiple apertures occurs, but its effect is feeble due to the short wavelength of X-rays. The grating converts X-ray tube radiation into multiple coherent sources; the wavefront is sampled to create a pattern inverse to the grating design. One-dimensional line gratings conventionally used in phase-contrast imaging allow only orientation-dependent analysis of structures, because only sensitivity in the direction, perpendicular to the grating lamellae, is possible [16]. Providing enhanced information on the material structure in several directions of two-dimensional gratings has been introduced $[9,12-14,16]$.

As in the single-shot phase-contrast imaging, the information is retrieved from the overlapped grating-object image, it is of great importance to ensure high quality and efficient performance of the grating. The sensitivity of the discussed single-shot imaging method scales with the period of the grating [9]. In order to sufficiently resolve the grating pattern, the camera pixel size should be equal to, or less than, one-third of the projected grating period [17]. This means that the detector's resolution sets the lower limit for the grating period. To achieve the highest possible resolution within a specific setup, the use of customized gratings is superior to the off-the-shelf grids. As it was reported previously [12], the gratings with greater symmetry may increase the efficiency of the wavefront sampling. A higher degree of periodicity of the grating structures improves the quality of the final image, thus, it is necessary to remove, or at least reduce to the minimum, the number of irregularities in the grating pattern. Another point to draw attention to is the profile of the grating structures. Due to the limitations imposed by the fabrication technique in use, usually, it is not a rectangular function. A possible slope of the grating sidewalls can introduce different absorption levels resulting in a blurring of the image, additional to the blurring by the X-ray tube focal spot size and point spread function of the camera. Thus, the improvement of gratings periodicity and shape can enhance the quality of the final image and possibly diminish the requirements on the $X$-ray tube and camera.

To evaluate the performance of the two-dimensional grating at the specific X-ray energy, one needs to study the wavefront sampling induced by it. The efficiency of wavefront modulation is a characteristic that directly influences the contrast of the image acquired by the detector. Here, by the 
efficiency of wavefront modulation (WFM), we mean the intensity variation between opaque and transparent grating areas as recorded by the camera. This can be expressed similarly to the visibility characteristic used in the interferometric methods [18]:

$$
\mathrm{WFM}=\frac{I_{t}-I_{o p}}{I_{t}+I_{o p}}
$$

where $I_{t}$ is the intensity, transmitted by the transparent structure, and $I_{o p}$ is the intensity, transmitted by the opaque structure. It is important to note that the values of $I_{t}$ and $I_{o p}$ vary significantly with $\mathrm{X}$-ray energy, and the same grating will perform differently at the different radiation energy. Moreover, as conventional sources, such as $\mathrm{X}$-ray tubes, are not monochromatic and emit energy in a certain spectrum, the ideal case of WFM $=1$ is not possible to attain in reality. Single-shot X-ray imaging has been demonstrated even with WFM $<0.5[4,15]$; however, increasing the efficiency of wavefront sampling can enhance the quality of the final image. WFM, being of relative value, can serve as a benchmarking measure for a particular grating to be used in a setup with the defined source and detector. To explore the possibilities of the single-shot imaging approach, one should achieve the highest wavefront modulation, keeping a high degree of periodicity for the gratings in use. In this regard, the objective of this work is to develop high-quality gratings of various periods to be used for customized materials science and medical applications at energies up to $25 \mathrm{keV}$.

\section{Materials and Methods}

UV lithography is one of the most available and widely-used microstructure fabrication techniques for patterning of periodic structures over a large area. It can provide gratings with high quality if the technological process is optimized for the required structure size regarding the diffraction limit of the resolution [19]. Each step of the manufacturing process requires optimization to eliminate the distortions of the predetermined pattern due to various phenomena, but greater attention must be paid to the fine-tuning of the exposure dose, which is directly related to the number of cross-linking reactions occurring in the photopolymer (photoresist) layer. Cross-linked areas form an intended pattern shape, which is subsequently used to serve as a mask for electroforming. Properties of the pattern, such as mechanical stability, homogeneity, and uniformity of the design (height and periodicity), directly depend on the photoresist performance during and after exposure. In the case of the negative photoresist, the exposure dose should be high enough to generate the cross-linking along the photoresist depth sufficient to adhere it to the substrate carrier and to avoid structure deflection followed by the pattern collapse due to insufficient stiffness. Overexposure, in turn, can result in significant distortion of the pattern shape due to diffusion of the photoactive components. Furthermore, overexposure can lead to cracking of the cross-linked areas due to photoresist shrinkage. The optimization of exposure dose requires a characterization of photoresist response to irradiation, which can be done by photoresist sensitivity curves.

\subsection{Sensitivity Curve for Negative Epoxy-Based Photoresist}

To determine the proper exposure parameters, we used sensitivity (contrast) curve procedure, which is used to assess the lithographic performance of photoresist [18]. A set of samples was prepared to carry out sensitivity curve procedure to determine the proper exposure parameters. A thin (few $\mathrm{nm}$ ) layer of hexamethyldisilazane was deposited on silicon wafers with a thickness of $525 \mu \mathrm{m}$ during substrate pretreating with heating up to $150{ }^{\circ} \mathrm{C}$ for $30 \mathrm{~min}$ to increase the adhesion of the photoresist to the wafer. Using different regimes to achieve layer thicknesses of $10 \mu \mathrm{m}, 15 \mu \mathrm{m}$, and $20 \mu \mathrm{m}$, the epoxy-based negative mr-X 10 photoresist (micro resist technology $\mathrm{GmbH}$, Berlin, Germany) was spin-coated on the wafers. Thicknesses higher than $20 \mu \mathrm{m}$ were not used for the sensitivity curve procedure, as in this case the dose is not uniformly distributed along the photoresist thickness due to absorption; the ratio of the bottom and top dose is not close to unity, which violates the basic assumption of the approach [18]. 
The soft bake was performed using a hot plate at the temperature of $95^{\circ} \mathrm{C}$ for $10 \mathrm{~min}$. Exposure was carried out with a mercury UV lamp. The stability and distribution of the total incident radiation power were controlled by Karl Suss UV intensity meter model 1000. The intensity value was $15 \mathrm{~mW} / \mathrm{cm}^{2}$ with $1 \mathrm{~mW} / \mathrm{cm}^{2}$ deviation from the center to the edge, the measurements were performed for the i-line of the mercury spectrum $(\lambda=365 \mathrm{~nm})$. The exposure was done using double-mask geometry: the lower mask was a Cr-quartz mask with alternating dark and light tone regions with a pattern of structure sizes from two up to $50 \mu \mathrm{m}$; the upper mask was a slit that allowed dividing the exposure area into 15 sectors that received a different dose with a constant step. The dose was tuned within a determined range by changing the exposure time. The exposure was carried out using vacuum contact alignment to eliminate diffraction effects: the wafer and the mask were placed in the vacuum chamber to ensure the absence of a gap between the mask and the photoresist layer.

The exposed samples were subjected to post exposure bake (PEB) in the oven for four hours with temperature changing from $70^{\circ} \mathrm{C}$ to $90^{\circ} \mathrm{C}$ and subsequent cooling to room temperature. A wet development of the exposed photoresist layers was carried out in propylene glycol monomethyl ether acetate in order to dissolve the unexposed area of $\mathrm{mr}-\mathrm{X} 10$ photoresist layers, and then the samples were rinsed in isopropanol alcohol. After development, the samples were air dried in a convection oven at $30^{\circ} \mathrm{C}$ and then the height of the formed micropattern was measured with a Tencor P-2 long scan profiler with an instrumental accuracy of $25 \AA$.

The results of the sensitivity curve measurements, which describe the remaining photoresist fraction as a logarithm function of the applied exposure dose, are shown in Figure 1. To approximate the data, we used a generalized logistic function fit according to the approach reported previously [18]. As one can see, the sensitivity curves for the 15 and $20 \mu \mathrm{m}$ photoresist layers are shifted towards higher exposure doses. This is due to the absorption introduced by the photoresist layer, which decreases the delivered bottom dose. All curves exhibited a similar slope, which can be associated with photoresist contrast. The contrast value is defined by:

$$
\gamma=\left(\log _{10}\left(\frac{D_{2}}{D_{1}}\right)\right)^{-1}
$$

where $D_{1}$ is the dose for which the remaining photoresist height is $10 \%$ of the original value, and $D_{2}$ is the dose for which the remaining photoresist height is $90 \%$ of the original value. The value of the contrast generally represents the response of the photoresist to radiation [18]. From the fabrication point of view, the most valuable information for a given patterning photoresist material is the optimal dose. By optimal dose, we mean the value that is sufficient to obtain a full cross-link of the photoresist. Optimal values were estimated from the sensitivity curve plateau, taking into account the pattern quality evaluated with an optical microscope (Figure 1). Defect-free patterns were obtained at the optimal dose value, which also served as a confirmation of properly-adjusted PEB and development time conditions. As it was noted previously, the thicknesses higher than $20 \mu \mathrm{m}$ could not be subjected to the sensitivity curve procedure; thus, the optimal values for such thicknesses were extrapolated from the data for lower thicknesses. 

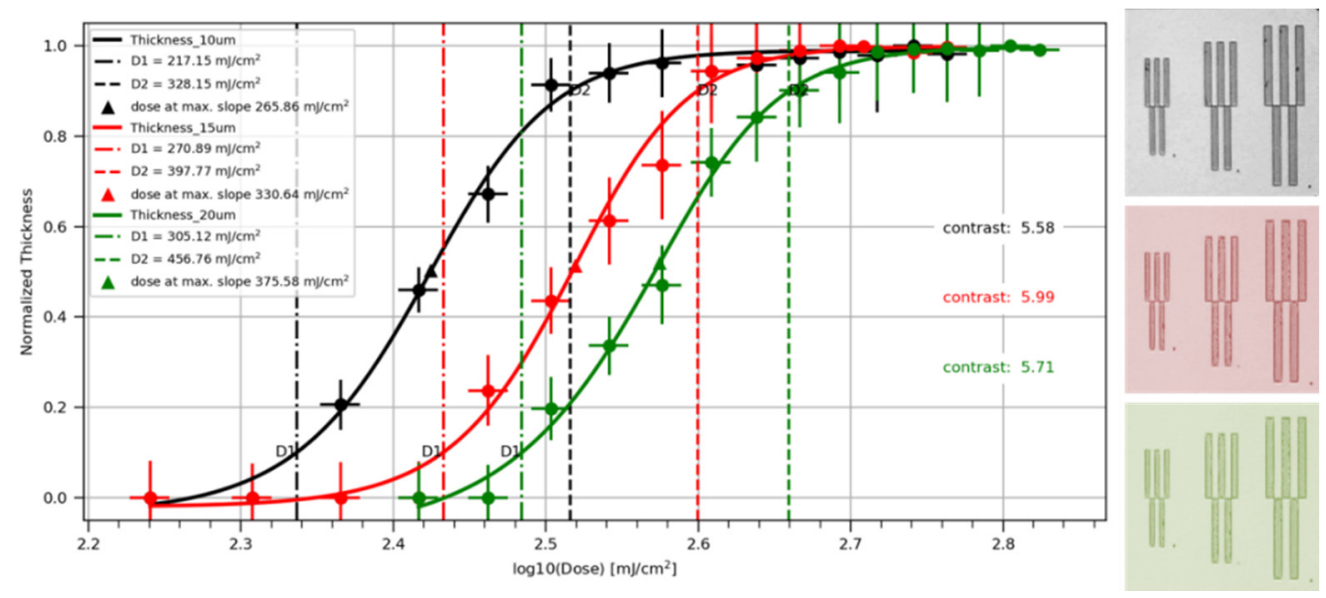

Figure 1. Sensitivity curves obtained for mr-X 10 photoresist layers of thickness: $10 \mu \mathrm{m}$ (black), $15 \mu \mathrm{m}$ (red), $20 \mu \mathrm{m}$ (green), and the respective colored pattern overview for $10 \mu \mathrm{m}$ (gray), $15 \mu \mathrm{m}$ (red), and $20 \mu \mathrm{m}$ (green). The curves were plotted using the approach reported in [18]. In the figure, contrast values for different curves are defined according to Equation (2). The dose at the maximum slope serves as a quantitative value for a comparison of the curves' shift.

\subsection{Grating Fabrication Process}

UV lithography for manufacturing of two-dimensional (2D) gratings was carried out using the process chain similar to the contrast curve sample preparation, but with several application-related changes. The main overview of the process is shown in Figure 2. Thinner silicon wafers were used for grating fabrication in order to achieve a higher wavefront modulation. The substrates were $200 \mu \mathrm{m}$ silicon wafers with few nanometers of $\mathrm{Ti}$ and $\mathrm{TiOx}$ to create a conductive contact layer for the concluding electroplating. Substrate cleaning was performed to guarantee that it was free of impurities and moisture. Oxygen plasma cleaning was employed with subsequent baking at $120^{\circ} \mathrm{C}$ and cooling to room temperature immediately before coating. Spin-coating of TI PRIME (MicroChemicals GmbH, Ulm, Germany) adhesion promoter was performed prior to photoresist application. TI PRIME forms a physically-bonded sub-monolayer of the active compound after solvent evaporation. A subsequent baking step of the primed substrate at $120^{\circ} \mathrm{C}$ chemically activated the adhesion promoter, thus forming the desired hydrophobic surface allowing the subsequent photoresist coating with improved wetting and adhesion between the TiOx and the photoresist. Immediately after the primed substrate baking was finished, spin-coating of the negative $\mathrm{mr}-\mathrm{X} 10$ photoresist was performed to achieve layers of $10 \mu \mathrm{m}, 20 \mu \mathrm{m}, 30 \mu \mathrm{m}$, and $40 \mu \mathrm{m}$ thicknesses. A soft bake to remove the solvent content from the sample was carried out at $95^{\circ} \mathrm{C}$ on hot plates for time periods from $10 \mathrm{~min}$ to $20 \mathrm{~min}$ depending on the photoresist layer thickness. 


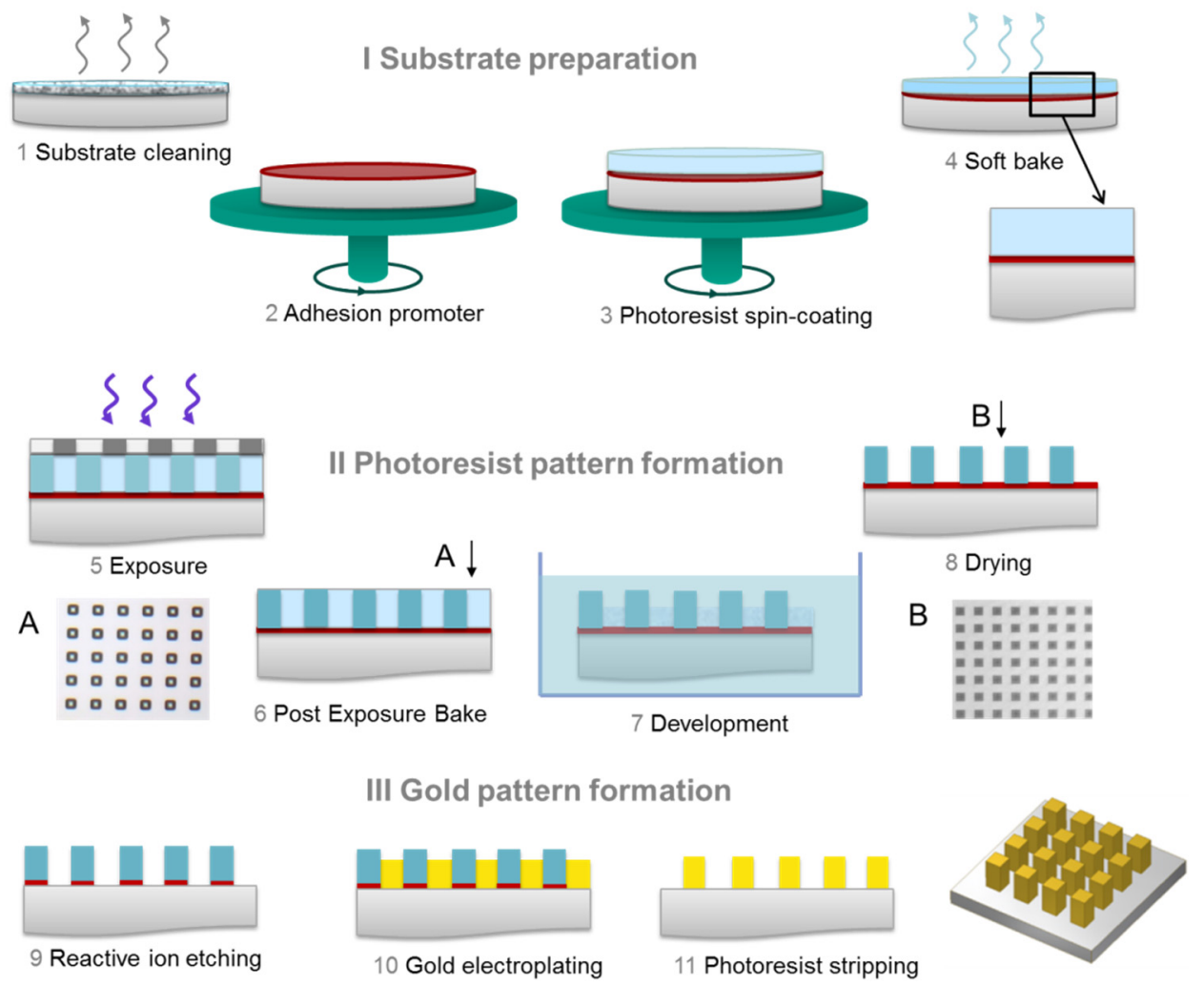

Figure 2. Fabrication process steps of UV LIGA: substrate preparation consisting of cleaning of the surface and applying the photoresist; photoresist pattern formation with exposure and development; and gold pattern formation by electroplating and stripping of the photoresist.

\subsubsection{Photoresist Pattern Formation}

The exposure of photoresist layers was performed using EVG mask aligner (EV Group, Sankt Florian am Inn, Austria) with $2.85 \mathrm{~mW} / \mathrm{cm}^{2}$ radiation intensity $\left(0.15 \mathrm{~mW} / \mathrm{cm}^{2}\right.$ deviation) using the filter for wavelengths shorter than $365 \mathrm{~nm}$. The photomask for exposure was a 5-inch Cr-Quartz mask purchased from Compugraphics (Jena, Germany) with four regions of different periods and shapes, and the area of each region was $1 \mathrm{~cm}^{2}$. The exposure was carried out in soft contact mode (no vacuum) in order to prevent the sticking of the photoresist layer to the mask. The exposure dose values are listed in Table 1. Theoretically, the optimal dose depends on the photoresist thickness, solvent, UV sensitivity, absorption in the exposure spectrum, power, and coherence of UV lamp, but for fabrication purposes it was empirically estimated using the sensitivity curve method. For thicknesses higher than $20 \mu \mathrm{m}$, the optimal dose values were extrapolated from the sensitivity curve data.

Post-exposure bake and development steps were the same as for the contrast curve sample set. Development times were adjusted according to the thickness of the photoresist layer. Optical microscope images of the gratings were obtained to perform a preliminary quality assessment of periodicity and homogeneity of the pattern. Sharp definition of the structures and a high degree of periodicity was observed for all periods for photoresist thickness up to $20 \mu \mathrm{m}$. However, for a photoresist thickness of $30 \mu \mathrm{m}$ and thicker, structures with a period of $10 \mu \mathrm{m}$ undergo undesirable crosslinking caused by the diffraction effect, which made square structures appear round. This resulted in structure size loss, as the final structures were electroplated using a structured photoresist layer as a mask for the conductive wafer surface. 
Table 1. Main characteristics of the fabricated gratings.

\begin{tabular}{|c|c|c|c|c|}
\hline $\begin{array}{l}\text { Photoresist } \\
\text { Thickness, } \mu \mathrm{m}\end{array}$ & Pattern Period, $\mu \mathrm{m}$ & Pattern Shape & Exposure Dose, $\mathrm{mJ} / \mathrm{cm}^{2}$ & Electroplated Thickness \\
\hline \multirow{4}{*}{10} & 50 & \multirow{3}{*}{ square } & \multirow{4}{*}{550} & \multirow{4}{*}{5} \\
\hline & 25 & & & \\
\hline & 10 & & & \\
\hline & 50 & round & & \\
\hline \multirow{4}{*}{20} & 50 & \multirow{3}{*}{ square } & \multirow{4}{*}{610} & \\
\hline & 25 & & & 15 \\
\hline & 10 & & & 9 \\
\hline & 50 & round & & 15 \\
\hline \multirow{4}{*}{30} & 50 & \multirow{3}{*}{ square } & \multirow{4}{*}{630} & \\
\hline & 25 & & & 22 \\
\hline & 10 & & & $(-)^{1}$ \\
\hline & 50 & round & & 22 \\
\hline \multirow{4}{*}{40} & 50 & \multirow{3}{*}{ square } & \multirow{4}{*}{650} & \\
\hline & 25 & & & 28 \\
\hline & 10 & & & $(-)^{1}$ \\
\hline & 50 & round & & 28 \\
\hline
\end{tabular}

${ }^{1}$ No structures could be obtained due to dramatic diffraction-induced shape distortion.

\subsubsection{Gold Electroplating}

The photoresist mask formed by means of UV lithography was filled with gold in an electroforming step after development. Electroplating current density and voltage were fixed and the duration time defines the amount of gold that should be plated on the wafer. This amount of gold was determined by the height of the designed structures, which is usually $75-80 \%$ of the photoresist layer thickness, and the area that should be electroplated. Gold electroforming was performed using a standard gold-sulfite electroplating bath for microstructure fabrication at a temperature of $55^{\circ} \mathrm{C}$. After electroplating, the photoresist was stripped using oxygen plasma.

The manufactured gratings and their main characteristics are listed in Table 1. Each grating has an area of $1 \mathrm{~cm} \times 1 \mathrm{~cm}$, as defined by the mask layout. However, larger areas (up to $5 \mathrm{~cm} \times 5 \mathrm{~cm}$ ) can be obtained within the same process chain if the respective mask layout is used.

\section{Results}

\subsection{Grating Quality Assessment with Scanning Electron Microscopy}

The grating quality evaluation was first performed with an optical and scanning electron microscope. The main quality control points were the uniformity of the grating pattern, the sharpness of structures edges, sidewall geometry, and quantitative characterization of the grating height, period, and duty cycle. The duty cycle (DC) in grating fabrication represents the ratio of the structure width to the period of the pattern. For the gold height from $5 \mu \mathrm{m}$ to $15 \mu \mathrm{m}$, it was possible to obtain reproducible structures with periods down to $10 \mu \mathrm{m}$; however, the shape of the structures was affected by diffraction in the corners of the square pillars. For gold structures higher than $20 \mu \mathrm{m}$, the electroplating of structures with a period of $10 \mu \mathrm{m}$ was not possible due to excessive cross-linking of the photoresist and significant distortion of the initial pattern shape.

An example of the grating of $25 \mu \mathrm{m}$ period and $28 \mu \mathrm{m}$ gold height with stripped photoresist is shown in Figure 3a. One can see that the grating pattern is clear and uniform; no structures are collapsed or detached. Each pillar of the grating is wider in the bottom and smaller on the top 
(Figure 4a), which is due to the interplay of a negative photoresist response, absorption, and diffraction effects occurring in the UV lithography. The duty cycle of the grating at the top and on the bottom is slightly different from the intended value. Analysis of SEM images has shown that, for the top of the grating, the duty cycle is 0.47 , and for the bottom, 0.58 . Thus, the average duty cycle is $0.52 \pm 0.05$. Note, that the analyzed grating has the highest aspect ratio (height $28 \mu \mathrm{m}$, structure size $12.5 \mu \mathrm{m}$ ) among fabricated gratings with sufficient quality (Figure 4a); the distortions observed for such structures are much less for the other fabricated gratings. From the application side such a distorted sidewall shape of the structures might slightly decrease the efficiency of the wavefront modulation, but, on the other hand, it provides structures with additional mechanical stability. The granular surface of the structures (see Figure 4) does not affect the quality of the wavefront modulation, as the gratings are to be used as an absorption mask.

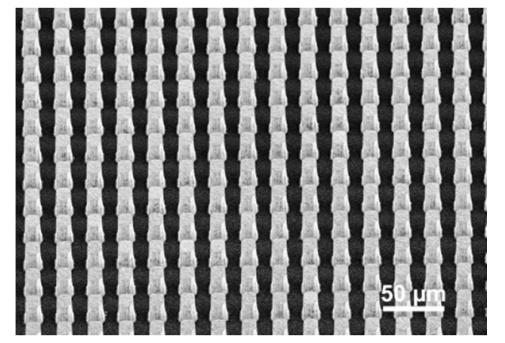

(a)

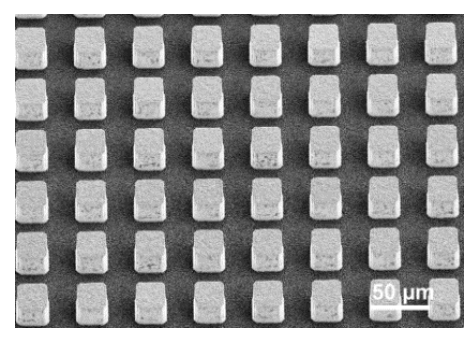

(b)

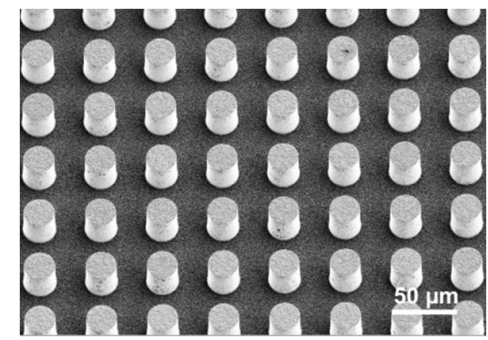

(c)

Figure 3. SEM overview images of two-dimensional X-ray gratings with $28 \mu \mathrm{m}$ gold height: (a) $25 \mu \mathrm{m}$ period pattern; (b) $50 \mu \mathrm{m}$ period square shape pattern; and (c) $50 \mu \mathrm{m}$ period round pattern.

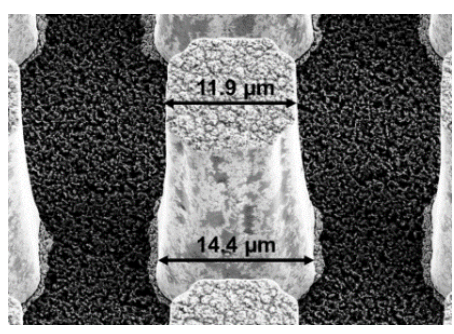

(a)

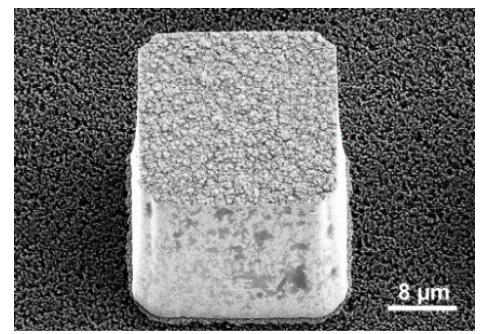

(b)

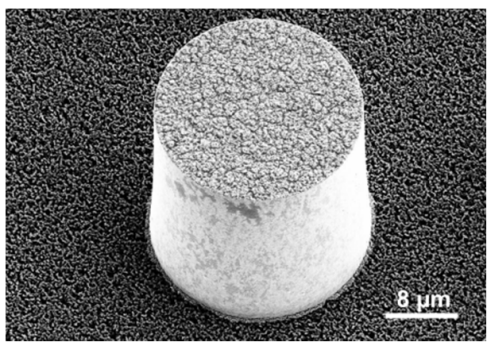

(c)

Figure 4. Close-up SEM images of two-dimensional X-ray gratings of $28 \mu \mathrm{m}$ gold height: (a) the square grating of the $25 \mu \mathrm{m}$ period; (b) the square grating of the $50 \mu \mathrm{m}$ period; and (c) the round pillar of the $50 \mu \mathrm{m}$ period.

As it was mentioned before, different shapes of the structures were tested to compare their final quality. The two-dimensional X-ray gratings of the $50 \mu \mathrm{m}$ period and $28 \mu \mathrm{m}$ gold heights are presented in Figure 3b,c. As one can see, the grating patterns of both shapes are clear and uniform, and the structures are well-defined and exhibit a high degree of periodicity. Single pillars of the square and round shapes are shown in Figure $4 \mathrm{~b}$,c. Both have a wider foundation and narrower top, but the deviation is smaller than the one presented in Figure 4a. For the square pillars, the top duty cycle is 0.49 and the bottom $\mathrm{DC}$ is 0.53 , which gives the average $\mathrm{DC}=0.51 \pm 0.02$. For the round pillar, the top duty cycle is 0.50 and for the bottom is 0.52 , which gives the average $D C=0.51 \pm 0.02$. The variation of the top and bottom sizes of the pillars is different for square and round shapes, but the average duty cycle remains the same. No period variation was observed in the SEM images. The values of DC for square and round structures imply that for smaller period one can profit from the decreased influence of diffraction in the patterning of round structures. 


\subsection{Analyzing the Grating Quality in a Radiography Setup}

We tested the developed two-dimensional grating structures using a radiographic setup at the TopoTomo beamline of the Karlsruhe synchrotron radiation facility (Karlsruhe, Germany). The measurements were performed at the energy of $8.5 \mathrm{keV}$ utilizing an optical system to introduce $9 \times$ effective magnification. The detector was a Neo sCMOS camera (Andor Technology Ltd., Belfast, UK) with an effective pixel size of $0.72 \mu \mathrm{m}$ and the field of view of $2560 \times 2140$ pixels. The grating was placed $8 \mathrm{~cm}$ from the detector. The tested gratings were gold square pillars of $15 \mu \mathrm{m}$ height with $10 \mu \mathrm{m}, 25 \mu \mathrm{m}$, and $50 \mu \mathrm{m}$ periods in both the vertical and horizontal directions, manufactured on the same 200- $\mu \mathrm{m}$-thick silicon substrate with an oxidized titanium layer. The average WFM introduced by the gratings according to the Equation (1) was 0.88 .

To test the robustness of the analysis algorithm we chose the gratings with several technologically-driven defects to impose challenges to the data interpretation. Our intention was to explore possibilities of decoupling the influence of typical fabrication issues using the data obtained from the projection image. The specimen has several defects in the photoresist pattern due to local adhesion issues, which were eliminated for other specimens by careful cleaning. As a consequence, the final pattern suffered from the overplating of gold on top of the photoresist layer, as shown in Figure 5a, diffraction-induced shape distortion, as represented in Figure 5b, and the impurity of the inter-structure areas and particle formation during electroplating, which can be seen in Figure 5c.

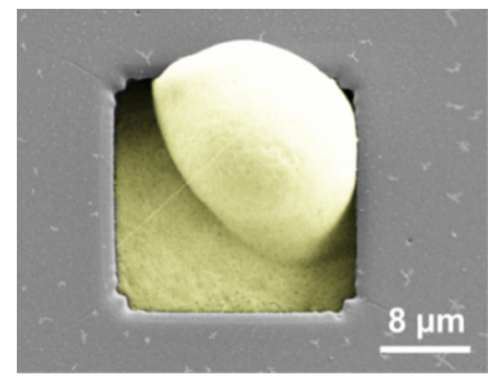

(a)

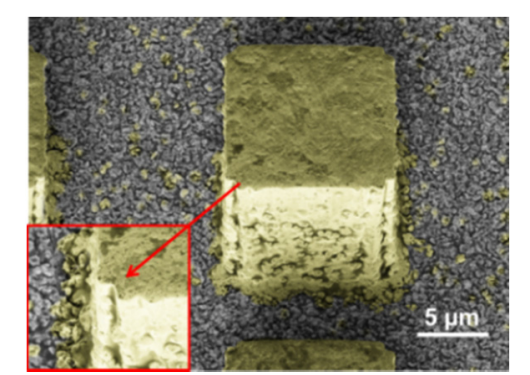

(b)

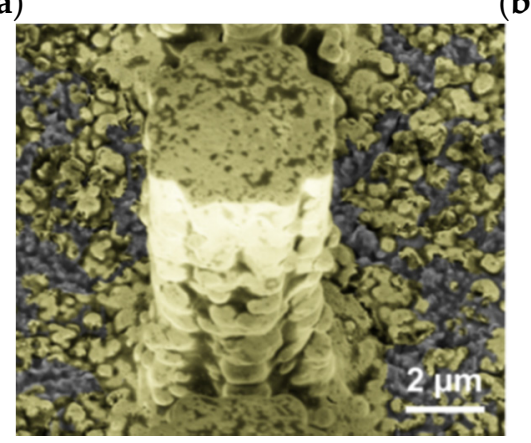

(c)

Figure 5. SEM images of the gratings chosen to test the robustness of the characterization algorithm by radiographic projection analysis: (a) the illustration of the gold overplating (grating before photoresist stripping); (b) illustration of pattern impurity and shape distortion caused by diffraction limit; and (c) large particle formation during electroplating.

Radiographic images were obtained with only the gratings in the beam path. The fragments of the images are shown in Figure 6c. Flat-field images without the gratings were obtained to correct the resulting image considering the beam shape and estimated detector noise. The exposure time for all acquired images was the same. As one can see in Figure 6a, there is a strong deviation of the background from the mean value and moreover, it has a highly inhomogeneous distribution over the whole detector area. The detector signal (recorded intensity) was composed of two Gaussians as shown in Figure 6b, which means that the illuminated detector area has two main intensity values (around 
7500 a.u. and 8500 a.u.). The mean value for the whole distribution was around 8400 a.u. Such an effect could be either due to a non-uniform beam or detector response. Considering the inhomogeneity of the background, special attention was paid to the normalization of the data. For each grating projection, the respective flat-field image was subtracted from the data and further correction by the absorption of the substrate was applied.

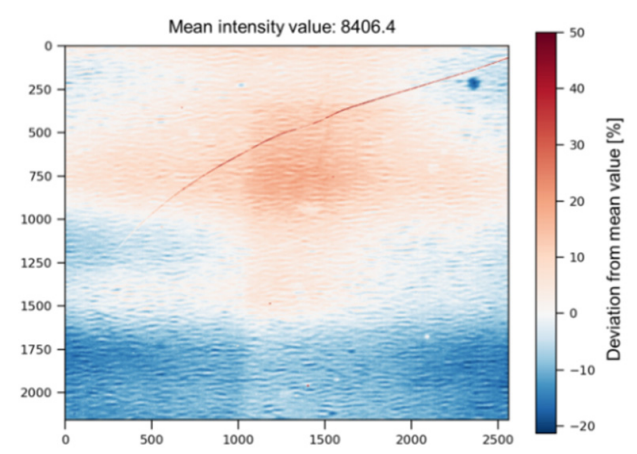

(a)

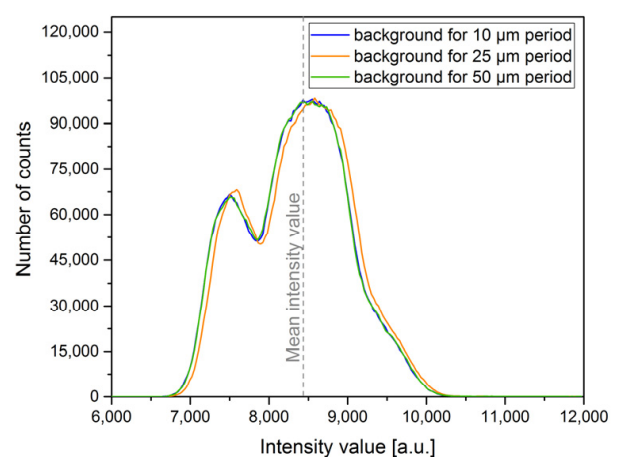

(b)

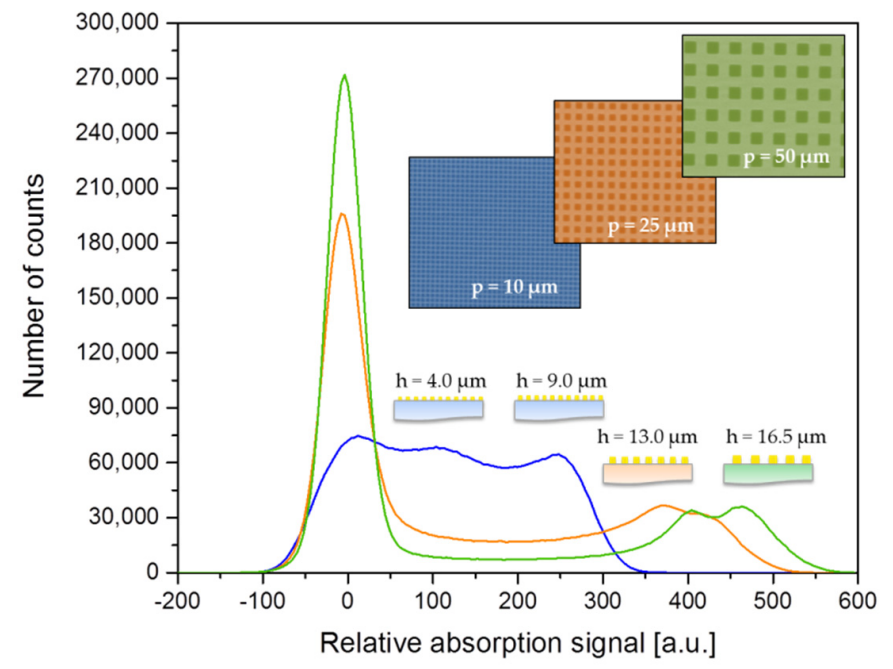

(c)

Figure 6. Radiographic image analysis: (a) flat-field image illustrating the deviation from the mean intensity value for the background; (b) illustration of the detector signal (recorded intensity) splitting in two Gaussians; and (c) the histogram of the relative absorption signal after subtraction of the background with cropped radiographic projection images for $10 \mu \mathrm{m}$ (blue), $25 \mu \mathrm{m}$ (orange), and $50 \mu \mathrm{m}$ (green) period square structures for visual representation.

Relative absorption signal histograms (i.e., the number of counts associated with a certain absorption level) were plotted by applying $3 \times 3$ median filters for pixel-noise removal to qualitatively characterize the intensity modulation introduced by the gratings. As one can see in Figure $6 c$, the intensity distribution peaks for gratings of periods $50 \mu \mathrm{m}$ and $25 \mu \mathrm{m}$ are clearly separated. However, there is still a splitting of the absorption signal peak around $400 \mathrm{a}$.u. for the $25 \mu \mathrm{m}$ period grating and around $430 \mathrm{a}$.u. for $50 \mu \mathrm{m}$ period grating. For the $10 \mu \mathrm{m}$ period, there was no clear separation of peaks observed, but, rather, an overlapping of at least three Gaussians centered at 0 a.u., 110 a.u., and 250 a.u. By comparison of the quantitative data regarding the height of gold structures obtained from the SEM images with absorption signal distributions, one can correlate the height of gold with the respective peak on the histogram. 


\section{Discussion}

To understand the data obtained from the measurements and evaluate the quality of the microstructure patterns over a large area, we correlated the grating defects and flaws with respective projection image distortions. In order to investigate possible generalized imperfections of the grating and its alignment in the setup, ideal data arrays were simulated, and their changes caused by the introduction of rotation and blurring were analyzed in terms of the absorption signal histograms. It is important to note that the proposed quality evaluation algorithm is giving useful information only for intrinsically periodic structures, such as gratings.

The main idea of the grating quality evaluation algorithm was to test the grating in a radiography setup to compare the intensity distribution obtained on the detector plane with the simulated intensity map from an ideal grating. By modifying the simulated ideal grating, one can estimate, quantitatively, the intensity modulation and characterize structural changes in the real grating. Using a simplified empirical model, an ideal grating was represented by a two-dimensional array of data with exactly the same dimension as the digital image obtained from the real data files acquired with the detector, taking into account the magnification. The array was filled with a constant value, and an intensity distribution histogram was obtained. This gave a discrete spectrum consisting of two peaks at absorption signals for the wafer (if normalized by the absorption level of the wafer, this peak is centered at zero) and the gold structures. The ratio of peaks is 1:3, as the gold structures cover one-third of the illuminated grating area in the simulated experiment.

When the arrays and associated intensity distribution histograms were obtained, the approximation of real gratings was achieved by adding a background function representing noise introduced by the experimental setup. This was done by introducing random fluctuations around the central value as detector noise (Gaussian distribution) or by using a real signal recorded by the detector in the experiment. The intensity distribution histogram with added noise is shown in Figure 7a. As the grating was supported by the substrate, it introduced a certain absorption, which decreased the signal-to-noise ratio and could also be treated as a contribution to the background. The detector noise was further estimated together with the contribution of 200- $\mu$ m-thick silicon absorption, thus, the histogram peaks were shifted in a way that the zero position corresponds to the radiation passing between the grating structures, and the second peak was placed at the absorption level of the gold structures, which was determined by the gold height.

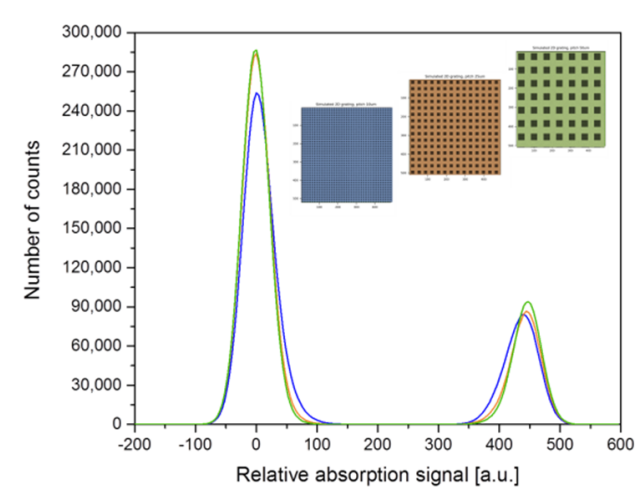

(a)

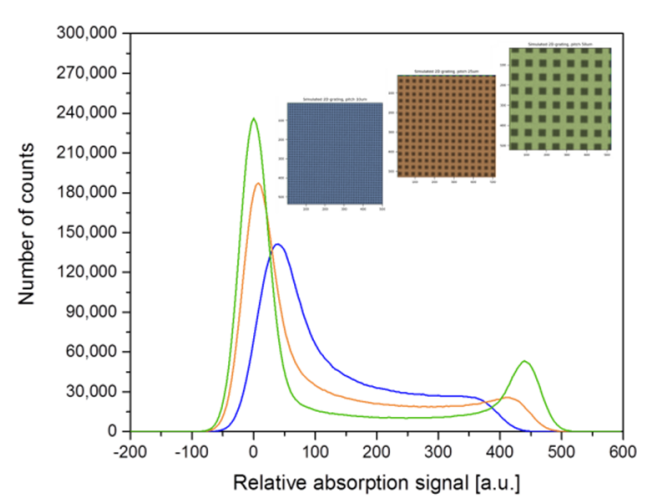

(b)

Figure 7. Simulated gratings intensity distribution histograms with added detector noise and cropped simulated data arrays with dimensions corresponding to the periods of $50 \mu \mathrm{m}$ (green), $25 \mu \mathrm{m}$ (orange), and $10 \mu \mathrm{m}$ (blue): (a) ideal data arrays; and (b) tilted at $-0.6^{\circ}$ and blurred data arrays. The simulation represents the influence of generalized pattern defects and was used to decouple them from the impact of local imperfections. 
However, in reality, not only does the presence of the detector noise decrease the efficiency of the intensity modulation, but also causes blurring by the imperfectly steep profile of structure sidewalls. Note that this manufacturing-driven blurring is independent of the blurring of the projected grating edges by the source focal spot size (focal spot size in case of an X-ray tube or lens focusing spot for a lens system). Despite the different origin, both result in blurring of the projected image, which is stronger for smaller periods: one can see that for the simulated grating with a $10 \mu \mathrm{m}$ period that the intensity distribution is not discrete anymore and peaks associated with the absorption of the wafer and the structures are merged and are not clearly distinguished, as can be seen in Figure $7 \mathrm{~b}$. The histogram shown in Figure $7 \mathrm{~b}$ has been obtained by varying the input parameters, such as the in-plane rotation of the grating and blurring of the image to match the experimental conditions and decouple the influence of generalized pattern defects from local imperfections. As one can see from the projection images shown in Figure 6c, there was a slight in-plane rotation of the grating present when the grating was placed in the holder. The rotation angle of 0.6 degrees was included in the empirical simplified model to better represent the acquired images and account for smoothening of the ripples in the histogram.

It is important to emphasize that the simulated data only included general distortions uniformly applied to the entire pattern and no local defect impact was considered. By comparison of the histogram obtained from the experimental data (Figure 6c) and the simulated one (Figure 7b), it is clearly seen that the pure pattern blurring cannot explain the distribution obtained for the gratings. In particular, the splitting of the peaks corresponding to gold absorption, especially evident for the $10 \mu \mathrm{m}$ period grating (Figure 8a), cannot be attributed to uniform effects present in the pattern but, rather, local defects of mainly random and rare appearance. One kind of such an effect is gold overplating, which causes the appearance of higher absorption levels. However, at the designed X-ray energy, the gold structures, with an average height of $15 \mu \mathrm{m}$, transmit only $0.5 \%$ of the incoming radiation [20]. This means that the gold structures higher than $14 \mu \mathrm{m}$ will not make a considerable effect on the intensity modulation. Overplated structures that contribute to the gold pillars higher than $20 \mu \mathrm{m}$ will not make any impact on the absorption signal histograms obtained from the measurements at the energy of $8.5 \mathrm{keV}$.

Another local effect present at the characterized gratings is pattern impurity due to partial gold deposition between the structures, which led to an overall decrease in transmission, thus, a decrease in intensity and additional broadening of the zero-centered peak. To analyze this effect in greater detail, the decomposition of real data histogram profiles into several Gaussian-shape signals has been performed. Examples for $10 \mu \mathrm{m}$ and $50 \mu \mathrm{m}$ period gratings are shown in Figure 8. It is evident in Figure $8 \mathrm{a}$ that an additional contribution of the signal at approximately 30 a.u. is present, corresponding to the gold height about $1 \mu \mathrm{m}$. This signal is attributed to the impurity of the pattern and contributed to the broadening of the zero-centered peak. The impurity of the pattern for the tested grating might be due to a slight undercut of the photoresist layer during reactive ion etching prior to electroplating. The effect was stronger for smaller periods, as observed with SEM (Figure 5b,c), in this case the gold nucleation points were placed closer to each other, and undesirable diffusion of $\mathrm{Au}^{+}$between the structures in the undercut area occurred. In turn, the contrast between absorbing and non-absorbing areas on the projected images was strongly decreased by this effect for smaller periods. For example, for the $10 \mu \mathrm{m}$ period grating, the highest pillars were about $9 \mu \mathrm{m}$ (Figure $5 \mathrm{c}$ ), thus, approximately $5 \%$ of the radiation was transmitted through such structures, and the transmission of the wafer carrier was reduced by the impurities of the pattern, resulting in a decreased intensity modulation.

However, not only did impurities contribute to the overlapping of the peaks on the histogram for $10 \mu \mathrm{m}$ period; as it was mentioned above, there is a third Gaussian centered at approximately 110 a.u. (the yellow dashed line in Figure 8a), which can be correlated with the gold height of about $4 \mu \mathrm{m}$ using information obtained with SEM. This implies that the gold height is not homogeneous and pillars of different height were obtained during the electroplating. However, for the $50 \mu \mathrm{m}$ period grating, the intensity modulation was highly efficient, resulting in clearly separated signals from gold structures 
and the transparent areas with the ratio of 1:3. Nonetheless, a splitting of the signal in two Gaussians is present, attributing to $14 \mu \mathrm{m}$ and $16 \mu \mathrm{m}$ heights. Another defect that could cause changes in the distribution of the absorption signal is the diffraction-caused shape distortion, which is prevailing for the $10 \mu \mathrm{m}$ period grating. This effect can be diminished by using a circular-shaped mask for UV lithography, as such a shape is less sensitive to diffraction effects.

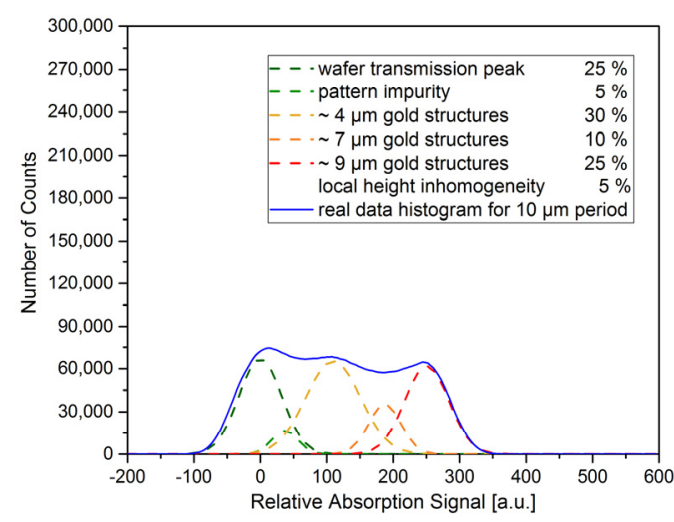

(a)

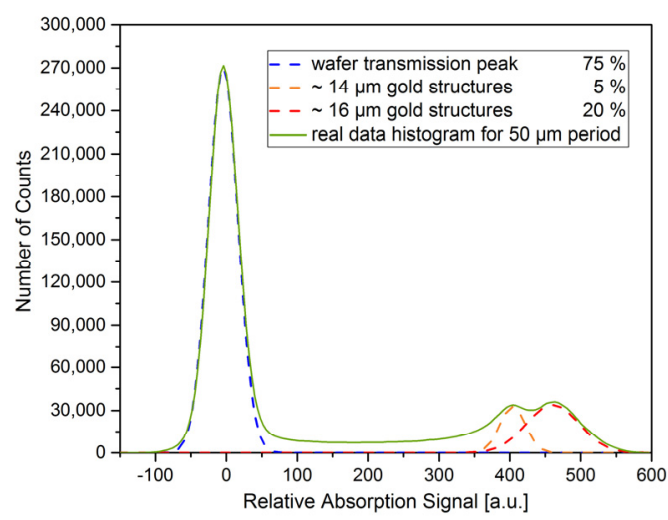

(b)

Figure 8. Real data absorption signal histogram decomposed into several Gaussian shape signals correlated to the different height of gold structures: (a) for a $10 \mu \mathrm{m}$ period grating (small Gaussians were treated as a local gold height inhomogeneity as their content in the entire profile was less than $2 \%$ each); and (b) for a $50 \mu \mathrm{m}$ period grating. In the legends, the percentage of the contribution to the signal was calculated from the areas of the Gaussians used for the approximation of the real data profiles.

\section{Conclusions}

In this work, the technological process for fabrication of two-dimensional X-ray gratings is described. The fabrication process is based on UV lithography and gold electroplating. Absorption gratings represented by periodic free-standing gold pillars with lateral structure sizes from $5 \mu \mathrm{m}$ to $25 \mu \mathrm{m}$ and heights from $5 \mu \mathrm{m}$ to $28 \mu \mathrm{m}$ have been manufactured. The gratings have shown a high degree of periodicity and defect-free patterns which enables their application for single-shot X-ray phase-contrast imaging. The gold height achieved in the manufacturing process absorbs more than $90 \%$ of X-ray radiation at the energy of $25 \mathrm{keV}$; for the transmission of $200-\mu \mathrm{m}$-thick silicon substrate at this energy the WFM is about 0.8 , which implies that the manufactured gratings can be used for single-shot imaging at energies up to $25 \mathrm{keV}$.

An algorithm allowing the qualitative and quantitative characterization of homogeneity and uniformity of the micropattern over a large active area involved in the imaging measurement was developed. The simulated images include general effects like blurring, detector resolution limitations, noise, and misalignments. However, they do not include any information on local defects, such as the diffraction-induced shape distortion, overplating, pattern impurity, mask defects transferred to the final grating, etc. Such defects are becoming critical for small periods, like $10 \mu \mathrm{m}$, as can be seen by comparing the real data histograms with the simulated data, while larger periods are less affected by the abovementioned defects and exhibit clear intensity modulation. From the evaluation of the grating structures in the radiographic setup, it follows that two-dimensional gratings with periods of $50 \mu \mathrm{m}$ and $25 \mu \mathrm{m}$ exhibit clear patterns and can be used for single-shot $X$-ray phase-contrast imaging at energies up to $25 \mathrm{keV}$. The gratings with $10 \mu \mathrm{m}$ period have multiple manufacturing-driven defects and require further quality improvement, for example, by using deep X-ray lithography or improved photoresist formulations. The evaluation of the grating quality will also be further improved, most probably introducing an image pattern recognition algorithm to be sensitive to small, localized defects.

The findings reported in this paper can be used for the improvement of image quality and broadening of applications for X-ray phase-contrast single-shot imaging towards materials 
characterization and medical imaging. The developed algorithm could also provide additional information on the grating quality by utilizing single projection for easier data interpretation during measurements of various objects.

Acknowledgments: This work was carried out with the support of the Karlsruhe Nano Micro Facility (KNMF), a Helmholtz Research Infrastructure at KIT; the Topo-Tomo beamline, located at the Karlsruhe synchrotron radiation facility of the KIT; the Karlsruhe School of Optics and Photonics (KSOP); and K. Daumiller (IKP, KIT).

Author Contributions: Margarita Zakharova and Danays Kunka planned the technology development for the radiography experiments. Margarita Zakharova fabricated the 2D gratings with the help of Heike Fornasier. Vitor Vlnieska tuned the negative photoresist to pattern the structures. Jürgen Mohr contributed with ideas to optimize the structure development. Margarita Zakharova, Danays Kunka, and Martin Börner characterized the 2D gratings. Margarita Zakharova, Danays Kunka, and Tomy dos Santos Rolo performed the experiments. Margarita Zakharova and Danays Kunka performed the data analysis. The manuscript was written by Margarita Zakharova and Danays Kunka with support from all the co-authors.

Conflicts of Interest: The authors declare no conflict of interest. The founding sponsors had no role in the design of the study; in the collection, analyses, or interpretation of data; in the writing of the manuscript; or in the decision to publish the results.

\section{References}

1. Wilkins, S.W.; Nesterets, Y.I.; Gureyev, T.E.; Mayo, S.C.; Pogany, A.; Stevenson, A.W. On the evolution and relative merits of hard X-ray phase-contrast imaging methods. Philos. Trans. R. Soc. A 2014, 372, 20130021. [CrossRef] [PubMed]

2. Pfeiffer, F.; Bench, M.; Bunk, O.; Kraft, P.; Eikenberry, E.F.; Brönnimann, C.; Grünzweig, C.; David, C. Hard-X-ray dark-field imaging using a grating interferometer. Nat. Mater. 2008, 7, 134-137. [CrossRef] [PubMed]

3. Weitkamp, T.; Diaz, A.; David, C.; Pfeiffer, F.; Stampanoni, M.; Cloetens, P.; Ziegler, E. X-ray phase imaging with a grating interferometer. Opt. Express 2005, 13, 6296-6304. [CrossRef] [PubMed]

4. Sato, G.; Kondoh, T.; Itoh, H.; Handa, S.; Yamaguchi, K.; Nakamura, T.; Nagai, K.; Ouchi, C.; Teshima, T.; Setomoto, Y.; et al. Two-dimensional gratings-based phase-contrast imaging using a conventional X-ray tube. Opt. Lett. 2011, 36, 3551-3553. [CrossRef] [PubMed]

5. Olivo, A.; Ignatyev, K.; Munro, P.R.T.; Speller, R.D. Noninterferometric phase-contrast images obtained with incoherent X-ray sources. Appl. Opt. 2011, 50, 1765-1769. [CrossRef] [PubMed]

6. Krejci, F.; Jakubek, J.; Kroupa, M. Single grating method for low dose 1-D and 2-D phase contrast X-ray imaging. J. Instrum. 2011, 6, C01073. [CrossRef]

7. Morgan, K.S.; Paganin, D.M.; Siu, K.W. Quantitative single-exposure X-ray phase contrast imaging using a single attenuation grid. Opt. Express 2011, 19, 19781-19789. [CrossRef] [PubMed]

8. Von Teuffenbach, M.; Koehler, T.; Fehringer, A.; Viermetz, M.; Brendel, B.; Herzen, J.; Proksa, R.; Rummeny, E.J.; Pfeiffer, F.; Noël, P.B. Grating-based phase-contrast and dark-field computed tomography: A single-shot method. Sci. Rep. 2017, 7, 7476. [CrossRef] [PubMed]

9. Wen, H.H.; Bennett, E.E.; Kopace, R.; Stein, A.F.; Pai, V. Single-shot X-ray differential phase-contrast and diffraction imaging using two-dimensional transmission gratings. Opt. Lett. 2010, 35, 1932-1934. [CrossRef] [PubMed]

10. Wu, B.; Liu, Y.; Rose-Petruck, C.; Diebold, G.J. X-ray spatial frequency heterodyne imaging. Appl. Phys. Lett. 2012, 100, 061110. [CrossRef]

11. Vittoria, F.A.; Kallon, G.K.N.; Basta, D.; Diemoz, P.C.; Robinson, I.K.; Olivo, A.; Endrizzi, M. Beam tracking approach for single-shot retrieval of absorption, refraction, and dark-field signals with laboratory $\mathrm{X}$-ray sources. Appl. Phys. Lett. 2015, 106, 224102. [CrossRef]

12. Bruza, P.; Panek, D.; Vrbova, M.; Fidler, V.; Rose-Petruck, C. Spatial frequency heterodyne imaging in soft X-ray water window. Appl. Phys. Lett. 2014, 104, 234101. [CrossRef]

13. Rand, D.; Derbak, Z.; Carlson, R.; Wands, J.R.; Rose-Petruck, C. X-ray Scatter Imaging of Hepatocellular Carcinoma in a Mouse Model Using nanoparticle Contrast Agents. Sci. Rep. 2015, 5, 15673. [CrossRef] [PubMed]

14. Rand, D.; Walsh, E.G.; Derdak, Z.; Wands, J.R.; Rose-Petruck, C. A highly sensitive x-ray imaging modality for hepatocellular carcinoma detection in vitro. Phys. Med. Biol. 2015, 60, 769-784. [CrossRef] [PubMed] 
15. Schunk, F.M.; Rand, D.; Rose-Petruck, C. Spatial frequency heterodyne imaging of aqueous phase transitions inside multi-walled carbon nanotubes. Phys. Chem. Chem. Phys. 2015, 17, 31237-31246. [CrossRef] [PubMed]

16. Kottler, C.; David, C.; Pfeiffer, F.; Bunk, O. A two-dimensional approach for grating based differential phase contrast imaging using hard X-rays. Opt. Lett. 2007, 15, 1175-1181. [CrossRef]

17. Bennett, E.E.; Kopace, R.; Stein, A.F.; Wen, H. A grating-based single-shot X-ray phase contrast and diffraction method for in vivo imaging. Med. Phys. 2010, 37, 6047-6054. [CrossRef] [PubMed]

18. Kunka, D.; Mohr, J.; Nazimov, V.; Meiser, J.; Meyer, P.; Amberger, M.; Koch, F.; Schulz, J.; Walter, M.; Duttenhofer, T.; et al. Characterization method for new resist formulations for HAR patterns made by X-ray lithography. Microsyst. Technol. 2014, 20, 2023-2029. [CrossRef]

19. Hibbs, M.S. System overview of optical steppers and scanners. In Microlithography Science and Technology, 2nd ed.; Suzuki, K., Smith, B.W., Eds.; CRC Press: Boca Raton, FL, USA, 2007; pp. 3-96, ISBN 9780824790240.

20. X-Ray Attenuation and Absorption Calculator. Available online: http://web-docs.gsi.de/ stoe_exp/web_ programs /x_ray_absorption/index.php (accessed on 25 November 2017).

(C) 2018 by the authors. Licensee MDPI, Basel, Switzerland. This article is an open access article distributed under the terms and conditions of the Creative Commons Attribution (CC BY) license (http:/ / creativecommons.org/licenses/by/4.0/). 